\title{
Viscosity and social cohesion in temporal lobe epilepsy
}

\author{
S M Rao, O Devinsky, J Grafman, M Stein, M Usman, T W Uhde, W H Theodore
}

Department of

Neurology, Medical

College of Wisconsin,

Milwaukee, Wisconsin SM Rao

Department of

Neurology, New York

University School of

Medicine, Hospital for

Joint Diseases, New

York, New York

O Devinsky

Medical Neurology

Branch, National

Institute of

Neurological

Disorders and Stroke,

Bethesda, Maryland

Cognitive

Neuroscience Section

J Grafman

Clinical Epilepsy

Section

W H Theodore

Section on Anxiety

and Affective

Disorders, Biological

Psychiatry Branch,

National Institute of

Mental Health,

Bethesda, Maryland

$M$ Stein

$T$ W Uhde

Department of

Medicine, Allegheny

General Hospital,

Pittsburgh,

Pennsylvania

M Usman

Correspondence to:

Dr Rao, Section of

Dr Rao, Section of

College of W isconsin, 9200

College of Wisconsin

Milwaukee, WI 53226, USA

Received 30 October 1990

and in final revised form

14 May 1991.

Accepted 22 May 1991

\begin{abstract}
Clinical case reports suggest that viscosity, the behavioural tendency to talk repetitively and circumstantially about a restricted range of topics, is common in patients with temporal lobe epilepsy (TLE). Such patients are also reported to exhibit heightened levels of social cohesion, the tendency to become interpersonally "clingy". This "sticky" interpersonal style may be particularly common in TLE patients with a left sided temporal lobe seizure focus. To test this hypothesis, self-report and observer rating scales were developed to assess both viscosity and social cohesion. Subjects consisted of patients with right, left, or bilateral temporal lobe seizure foci, absence or primary generalised tonic-clonic seizures, psychiatric controls (panic disorder patients), and normal controls. Elevations on the viscosity scale were observed primarily in TLE patients with left or bilateral seizure foci. Viscosity scores also correlated with seizure duration and left handedness. No group differences were observed on the social cohesion scale. These findings are consistent with the hypothesis that viscosity results from subtle interictal language disturbances, although other pathogenetic mechanisms are discussed.
\end{abstract}

Diverse and unusual personality traits have been attributed to patients with temporal lobe epilepsy (TLE).$^{1-3}$ One such trait has been termed "viscosity". ${ }^{34}$ This trait has had two meanings in the epilepsy literature: $:^{356}$ stickiness of thought processes or enhanced interpersonal adhesiveness, also referred to as increased social cohesion. Such patients are often described as talking repetitively and circumstantially, sticking to restricted topics, and having difficulty in breaking off conversations. Increased social cohesion is characterised by a tendency to form clinging relationships with family members and close friends. Empirical evidence to support the observations noted in these clinical case reports has been provided by Bear et al. ${ }^{5}$ In a blinded comparison of TLE patients and mixed psychiatric control patients, they found that viscosity, rated from structured psychiatric interviews, was the best of 14 behavioural traits at discriminating between the two groups. Our understanding as to why some TLE patients apparently develop vis- cosity and increased social cohesion is lacking. Animal experiments have shown that discrete limbic lesions alter social awareness and increase desire to maintain contacts with other animals of the same or different species. ${ }^{7-9}$ Specifically, rats with septal lesions exhibit strong tendencies to remain in contact with each other in an open field and if left alone appear fearful and will actively approach cats. ${ }^{9}$ Alternatively, several investigators have observed high rates of language disturbance in TLE patients, particularly those with dominant hemisphere epileptic foci. ${ }^{1011}$ Mayeux et $a l^{11}$ reported a significant correlation between impaired naming and circumstantial speech, suggesting that viscosity may be caused by subtle interictal linguistic disruption rather than a personality trait. One might predict, therefore, a greater likelihood of viscosity in TLE patients with left as opposed to right temporal lobe foci. Hoeppner et al $^{12}$ administered the "cookie theft" card of the Boston diagnostic aphasia examination ${ }^{13}$ to patients with temporal lobe, generalised, and simple partial epilepsy, and controls. Taped responses were blindly rated. All four patients with verbose and circumstantial responses had left temporal foci. With the Bear-Fedio ${ }^{1}$ selfreport viscosity scale, Brandt et al ${ }^{14}$ also found a laterality effect-that is, left TLE patients exhibited greater viscosity than right TLE patients; but other investigators, using this same scale, have not found a laterality effect. ${ }^{15}$ These contradictory findings may have resulted from the small number of items (five) that compose the Bear-Fedio viscosity scale. Furthermore, the scale fails to discriminate repetitive circumstantial speech from increased social cohesion. Possibly laterality effects, when observed, are associated with one of these behaviours and not the other.

We developed comprehensive self-report and rater scales for both viscosity, defined strictly as repetitive and circumstantial speech, and social cohesiveness. We embedded these two scales within a longer test, which provides additional scales for paranoid tendencies and a defensive response style (that is, the L scale from the Minnesota multiphasic personality inventory). The scales were administered to patients with left, right, or bilateral TLE, patients with absence or generalised epilepsy, a psychiatric control group consisting of panic disorder patients, and normal controls. To gain a better understanding of possible aetiological factors 
associated with these behavioural traits, we correlated test scores with seizure variables (duration, frequency) and handedness.

\section{Material and methods \\ Subjects}

A total of 118 subjects participated in this study: 18 with temporal lobe epilepsy-left hemisphere focus (TLE-L), 21 with temporal lobe epilepsy-right hemisphere focus (TLER), 19 with temporal lobe epilepsy-bilateral foci (TLE-B), 12 with absence or generalised seizures (ABS-GEN), 25 with panic disorders (PANIC) serving as a psychiatric control group, and 23 normal controls (NORMAL). In 53 of the 58 TLE patients, the seizure focus was identified by prolonged inpatient video-EEG monitoring and recording of ictal events. TLE$B$ patients had bilateral, independent, interictal epileptiform discharges or had non-lateralised seizure onsets during video-EEG monitoring. For the five TLE patients who did not undergo video-EEG monitoring, lateralisation was based on findings of interictal epileptiform activity on at least two EEGs and no evidence of contralateral, interictal epileptiform activity. Patients with primary generalised epilepsy, all of whom continued to have absence seizures at the time of study, were diagnosed with at least two EEGs which were consistent with bilaterally synchronous and symmetrical 34.5 $\mathrm{Hz}$ spike-and-wave complexes. No clinical features of partial seizures or evidence of focal epileptiform activity were noted in the ABSGEN group. We excluded seizure patients with well-defined brain injury or disease (ie patients with temporal lobe resections, tumours, stroke, severe head trauma). Patients with histories of substance abuse or mental retardation were also excluded. The diagnosis of panic disorder was based on DSM-III-R ${ }^{16}$ criteria. The normal control group was recruited by posted advertisement; potential subjects were excluded if they had a history of neurological or psychiatric disorders. Handedness was assessed with a modified Edinburgh scale. ${ }^{17}$ Demographic and seizure statistics are presented in the table. Significant $(p<0.05)$ group differences were observed for age (NORMAL and PANIC > TLE-R), education (NORMAL > TLE-R), and occupational status (NORMAL > all seizure groups). To correct for these demographic mismatches, all statistical group comparisons with behavioural data (see results below) were performed with the analysis of covariance with age, education, and occupational status serving as covariates.
Among the seizure patients, no significant group differences were observed in duration or frequency of seizures. TLE-L and TLE-B patients were more likely to be left handed or ambidextrous $(22 \%$ and $26 \%$, respectively) than the other four groups combined $(10 \%)$.

\section{Behavioural Measures}

The questionnaires were specifically designed for this study. All subjects completed a 32 item true-false questionnaire (see appendix) that yielded scores to four summary scales: viscosity (nine items), social cohesion (13 items), paranoia (five items), and five items from the lie scale of the MMPI. Internal consistency (Cronbach's alpha ${ }^{18}$ ) of the scales ranged from $r=0.55$ (social cohesion) to $r=0.61$ (paranoia); testretest reliability was not evaluated. Similar traits were also rated by a close relative (spouse, parent, sibling) or friend with a 24 item truefalse questionnaire (see appendix): viscosity (11 items), social cohesion (seven items), and paranoia (six items). Ratings were obtained for 100 of the 118 subjects. A third of the raters were friends. Internal consistency ranged from $\mathbf{r}=0.61$ (social cohesion) to $\mathbf{r}=\mathbf{0 . 8 4}$ (viscosity). Combining the groups, the selfreport and rater versions of each scale were found to be significantly correlated: viscosity $(\mathrm{r}=0.34, \quad \mathrm{p}<0.001)$, social cohesion $(\mathrm{r}=0.34, \mathrm{p}<0.001)$, and paranoia $(\mathrm{r}=0.41$, $\mathrm{p}<0.001$ ).

\section{Statistical analysis}

For each scale, group differences were analysed with analysis of variance. Significant group effects were then subjected to Tukey B posthoc analyses to determine which groups were significantly different from each other. Correlational analyses were performed to assess the relation between seizure variables and scale scores. Given the large number of analyses performed, a more conservative alpha level $(p=0.01)$ was selected. In the light of the exploratory nature of the study, however, nonsignificant trends $(0.01<\mathrm{p}<0.05)$ are also reported.

\section{Results}

No significant group differences $(p>0 \cdot 10)$ were observed on the social cohesion or paranoia scales for either method of administration (self-report or rater); likewise, no differences were observed on the self-report lie scale. The figure presents the mean viscosity scores along with the standard error of the mean for each group. For the self-report scale,

Table Demographic and seizure variables

\begin{tabular}{|c|c|c|c|c|c|c|c|}
\hline & $\begin{array}{l}\text { Sex } \\
(F / M)\end{array}$ & $\begin{array}{l}\text { Age } \\
\text { (years) }\end{array}$ & $\begin{array}{l}\text { Education } \\
\text { (years) }\end{array}$ & $\begin{array}{l}\text { Occupation } \\
\text { (0-4 pts) }\end{array}$ & $\begin{array}{l}\text { Left-handed } \\
\text { No }(\%)\end{array}$ & $\begin{array}{l}\text { Seizure } \\
\text { duration } \\
\text { (years) }\end{array}$ & $\begin{array}{l}\text { Seizure } \\
\text { frequency } \\
\text { (per year) }\end{array}$ \\
\hline $\begin{array}{l}\text { TLE-left focus } \\
\text { TLE-right focus } \\
\text { TLE-bilateral } \\
\text { ABS-GEN } \\
\text { Panic disorder } \\
\text { Normal controls }\end{array}$ & $\begin{array}{l}8 / 10 \\
9 / 12 \\
12 / 8 \\
9 / 3 \\
22 / 3 \\
12 / 11\end{array}$ & $\begin{array}{l}33 \cdot 1 \\
29 \cdot 1 \\
33 \cdot 7 \\
28 \cdot 6 \\
37 \cdot 6 \\
37 \cdot 8\end{array}$ & $\begin{array}{l}14 \cdot 1 \\
12 \cdot 5 \\
14 \cdot 3 \\
13 \cdot 7 \\
14 \cdot 2 \\
15 \cdot 9\end{array}$ & $\begin{array}{l}1.4 \\
1.6 \\
1.6 \\
1.5 \\
2.1 \\
2.7\end{array}$ & $\begin{array}{l}4(22) \\
2(10) \\
5(26) \\
1(9) \\
4(16) \\
1(4)\end{array}$ & $\begin{array}{l}19 \cdot 3 \\
18 \cdot 7 \\
19 \cdot 1 \\
19.5\end{array}$ & $\begin{array}{l}27 \cdot 3 \\
36 \cdot 0 \\
24 \cdot 0 \\
61 \cdot 7\end{array}$ \\
\hline
\end{tabular}

TLE $=$ temporal lobe epilepsy. 
Figure Mean self-report and rater scores on the viscosity scale for left, right, and bilateral temporal lobe epilepsy patients (TLE-L, TLE$R$, and $T L E-B$, respectively), patients with absence or generalised seizures ( $A B S-G E N)$, panic disorder patients (PANIC), and normal controls (NORMAL). Error bars $=$ standard error of mean.

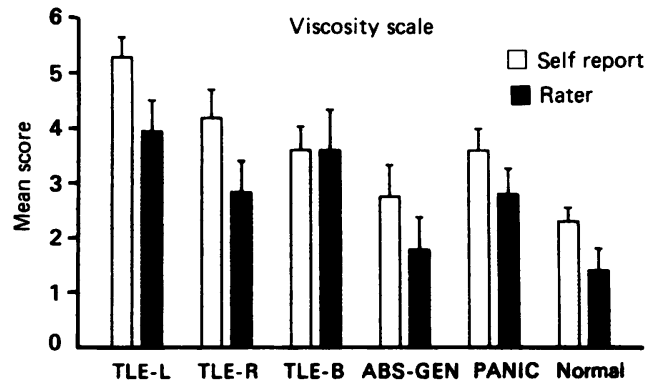

a significant overall group effect was observed $(\mathrm{F}[5,104]=4 \cdot 7, \quad \mathrm{p}=0.001) . \quad$ Post-hoc analyses indicated a significantly higher viscosity score for the TLE-L group compared to the ABS-GEN group and the normal and panic disorder control groups. Significant group differences were also observed for the relative or friend ratings of viscosity $(F[5,86]=3 \cdot 3$, $\mathrm{p}=0.009$ ). Post-hoc analyses indicated significantly higher viscosity scores in the TLE-L and TLE-B groups compared to the ABS-GEN and NORMAL groups. Significant correlations were observed between viscosity scale self-report scores and duration of seizures for the TLE-L group $(r=0.65, p<0.005)$. A marginally significant correlation was also found between duration and self-report viscosity in the TLE-B group $(r=0.50$, $p<0.05$ ). Seizure frequency did not correlate with viscosity scores for any of the groups. Likewise, seizure duration and frequency did not correlate with scores on the social cohesion, paranoia, or lie scales for any of the groups. To assess the possible role of handedness and viscosity, we compared the scores of right and left handed subjects on the relative or friend rating scales. The left handed TLE-L patients $(n=4)$ had higher viscosity scores than right handed TLE-L patients $(n=14)$, although the group difference was only marginally significant $(F[1,16]=6.8, p=0.02)$. No other group comparisons were significant.

\section{Discussion}

Our results suggest that viscosity, as measured by our self-report and rater scales, is a specific behavioural trait more commonly observed in epileptic patients with left-sided or bilateral temporal lobe seizure foci. Unlike those reported by Brandt et al ${ }^{14}$ our patients with generalised seizures exhibited significantly less viscosity on our self-report and rater scales than the TLE patients. This indicates that viscosity is not necessarily associated with having epilepsy. Mayeux et al ${ }^{11}$ previously suggested that viscosity is caused by deficits in language (such as anomia) or verbal memory or both in association with dominant hemisphere brain involvement. In contrast, Hoeppner et $a l^{12}$ found that language and memory test performance was no more impaired in their verbose TLE-L patients than in their TLE-L patients not exhibiting verbosity, suggesting that other factors may contribute to viscosity in TLE patients. Patients with anomia caused by structural lesions such as stroke do not typically display viscosity. Thus anomia and language dysfunction may be necessary, but not sufficient, conditions for the development of epilepsy-related viscosity.

As an alternative hypothesis, we speculate that viscosity is caused by a critical deficit in the TLE patients' ability to perceive social cues in conversation. These cues may include posture, facial expressions, and eye contact, in addition to linguistic communication. During normal conversation, participants assume mutual responsibility for assuring that what is said has been heard and understood before the conversation proceeds. ${ }^{1920}$ TLE patients with viscosity may fail to perceive that the listener has understood what was said, resulting in speech repetition or disjointed conversational switches. In addition, mental slowing, which is common in epilepsy patients partly due to seizure medications, may contribute to the perception of viscosity by observers. Future studies, with recently developed methods for assessing conversational communication, ${ }^{2021}$ may shed light on viscosity in TLE patients.

Not all of our TLE-L and TLE-B patients exhibited signs of viscosity. Scores on the viscosity scale correlated with duration of the seizure disorder in our left and bilateral TLE patients. Hoeppner $e a^{12}{ }^{12}$ also noted that their four verbose patients with left TLE had longer seizure durations than their five non-verbose patients with left TLE. These findings suggest that viscosity develops in adults with TLE-L after years of seizure activity. Alternatively, TLE patients with viscosity may have more extensive dominant hemisphere damage than patients without viscosity and, as a consequence, are more likely to develop seizures at an earlier age. We did not find a significant correlation between age at seizure onset and viscosity, thereby supporting the former "duration" hypothesis. Our TLE-L and TLEB patients had a greater proportion of left than right handers, and the left handers were more likely to exhibit viscosity. We speculate that left handedness is associated with early dominant hemisphere brain damage, so-called pathological left handedness, ${ }^{22}$ resulting in abnormal development of linguistic communication skills.

Our study is the first to assess viscosity separately from its related personality trait, social cohesion. Our data do not support the hypothesis that social cohesion is more common in TLE patients. Alternatively, our scale, as currently designed, may simply not be sensitive to the trait of social cohesiveness. Further reliability and validity research is needed to determine the utility of our scales in future neurobehavioural investigations of TLE patients.

Presented in part at the 18th Annual Meeting of the International Neuropsychological Society, Kissimmee, Florida, USA, February 1990.

\footnotetext{
Appendix

1 Most any time I would rather sit and daydream than do 1 Most any time 1

2 I have never told a lie in my whole life $[L-t]$

3 I prefer to pass by old friends or people I know but have not seen for a long time, unless they speak to me first [ $S C$-f]
} 
4 I sometimes keep on at a thing until others lose their patience with me $[V-t\rangle$

5 Things have never gone my way; I rarely get lucky breaks $[P-t]$

6 I love to go to parties, even if I know only one or two other people $[S C-t]$

7 I sometimes worry that people are talking about me in a "not so nice" way $[P-t]$

8 I need more time to myself than most people do $[S C-f]$

9 My fingernails sometimes burn and tingle $[L-t]$

10 Most people are boring; they talk about trivia and waste my 10 Most people

11 I have never been embarrassed [ $L-t$ ]

11 The never been embarrassed $[L-t]$ / 12 I always prefer to be in the company of at least one other

person $[S C-t]$

14 I would almost always prefer a long conversation with a friend than sit with a friend watching a beautiful sunset $[V-t]$ $15 \mathrm{My}$ phone conversations are usually ended by the person I am talking to $[V-t]$

16 I hate to leave anything unfinished $[V-t]$

17 I often get pain and an itching sensation right behind my kneecaps $[L-t]$

18 Sometimes I have to tell people several times to give them details (to make sure they get it right) $/ V-t$

19 I am basically a loner $[S C$ - $f$ ]

20 I often worry that someone has done something wrong or harmful to me $[P-t]$

21 Even when I am with people I feel lonely much of the time 21 Even
[SC-f]

22 I hate to say goodbye $\mid V-t$

23 People tend to interrupt me when I'm trying to make a point $\lceil V-t]$

24 I don't like to be alone for more than an hour or two [SC- $t$ ]

25 I dislike people around me / $S C$ - $f$ ]

26 I like to poke my nose into other people's business [ $S C-t$ ]

27 I have many friends $[S C-t$ ]

28 People tend to take advantage of me $[P-t]$

29 I enjoy listening to lectures or speeches by politicians [SC-t]

30 If asked for my opinion, I usually can find something more interesting to say than other people $[V-t]$

31 Sometimes my mind gets stuck on one idea and I cannot make a decision or do anything $[V-t]$

32 I am often suspicious of what other people are thinking or doing, that it may be harmful to me $[P-t]$

Rater scale
$1 \mathrm{He}$ (she) can talk your ear off $/ V-t]$

$2 \mathrm{He}$ (she) enjoys his (her) own company more than that of other people $[S C-f]$

3 When he (she) gets involved in a controversial debate or discussion, he (she) goes on and on $/ V-t$

$4 \mathrm{He}$ (she) often thinks that "life has been unfair to me" [P-t

$5 \mathrm{He}$ (she) is very dependent and always needs someone else around $/ S C$ - $t$.

$6 \mathrm{He}$ (she) is never brief and to the point; in contrast, he (she) seems to spend forever circling the point $[V-t]$

$7 \mathrm{He}$ (she) gets stuck on a topic and can't move on $[V-t]$

$8 \mathrm{He}$ (she) is basically a loner $/ S C$-f $/$

$9 \mathrm{He}$ (she) sometimes worries that people are talking about him (her) in a "not so nice way" [ $P-t]$

10 When I have a phone conversation with him (her), I always find that $I$ am the one who wants to get off first $/ V-t)$

$11 \mathrm{He}$ (she) is not very talkative $[V-f]$

$11 \mathrm{He}$ (she) is not very talkative $/ V-f /$
$12 \mathrm{He}$ (she) is not sensitive to hints that sometimes I need my $12 \mathrm{He}$ (she) is not

"own space" $|V-t|$ $13 \mathrm{He}$ (she) often worries that something wrong or harmful was done to him (her) $/ P-t$

14 Saying goodbye to him (her) can take forever $[V-t]$

$15 \mathrm{He}$ (she) is antisocial and doesn't like to be around people SC-f]

$16 \mathrm{He}$ (she) would prefer to pass by an old friend that he (she) hasn't seen for a long time, unless the friend was to speak to him (her) first / $S C$-f /

$17 \mathrm{He}$ (she) believes that people take advantage of him (her)

$18 \mathrm{He}$ (she) complains that most people are boring; he (she) prefers his (her) own company / $S C$-f /
$19 \mathrm{He}$ (she) never wants to go to parties or other social events [SC-f]

$20 \mathrm{He}$ (she) often repeats the same details over and over again

[ $21 \mathrm{He}$ ] (she) tends to be overly suspicious of things $[P-t]$

22 It seems as though he (she) can never say goodbye (that is, his (her) "goodbyes" are never brief) $[V-t]$

$23 \mathrm{He}$ (she) can get preoccupied and keep bringing up minor $23 \mathrm{He}(\mathrm{she})$ can get preoccupied and keep bringing up min
events which other people would let pass and forget $[V-t]$ $24 \mathrm{He}$ (she) occasionally worries that people are "out to get him (her)" $[P-t]$

$\mathrm{SC}=$ social cohesion; $\mathrm{V}=$ viscosity $\mathrm{P}=$ paranoia $\mathrm{L}=$ lie; $t=$ true; $f=$ false.

1 Bear D, Fedio P. Quantitative analysis of interictal behavior in temporal lobe epilepsy. Arch Neurol 1977;34:454-67.

2 Glaser GH. The problem of psychosis in psychomotor temporal lobe epileptics. Epilepsia 1964;34:271-8.

3 Blumer D. Temporal lobe epilepsy and its psychiatric significance. In: Bensic aspects of neurologic
Inc 1975:171-97.

4 Geschwind N. Pathogenesis of behaviour change in temporal lobe epilepsy. Res Pub Ass Nerv Ment Dis 1983;61:355-70.

5 Bear D, Levin K, Blumer D, Chetham D, Tyder J. Interictal behaviour in hospitalised temporal lobe epileptics: Relationship to idiopathic psychiatric synd
Neurol Neurosurg Psychiatry 1982;45:481-8.

6 Bear DM. Temporal lobe epilepsy-a syndrome of sensorylimbic hyperconnection. Cortex 1979;15:357-84.

7 Kolb B, Nonneman AJ. Frontolimbic lesions and social behavior in the rat. Physiol Behav 1974;13:637-43.

8 Glendenning KK. Effects of septal and amygdaloid lesions on social behavior of the cat. J Comp Physiol Psychol 1972;80:199-207.

9 Meyer DR, Ruth RA, Lavond DG. The septal social cohesiveness effect: its robustness and main determinants. Physiol Behav 1978;21:1027-9.

10 McIntyre M, Pritchard PB, Lombroso CT. Left and right temporal lobe epileptics: A controlled investigation of some psychological differences. Epilepsia 1976;17:377-86.

11 Mayeux R, Brandt J, Rosen J, Benson J. Interictal memory and language impairment in temporal lobe epilepsy. Neurology 1980;30:120-5.

12 Hoeppner JB, Garron DC, Wilson RS, Koch-Weser MP. Epilepsy and verbosity. Epilepsia 1987;28:35-40.

13 Goodglass $\mathrm{H}$, Kaplan $\mathrm{E}$. The assessment of aphasia and related disorders. Philadelphia: Lea and Febiger, 1983.

14 Brandt J, Seidman LJ, Kohl D. Personality characteristics of epileptic patients: A controlled study of generalized and temporal lobe cases. J Clin Exp Neuropsychol 1985;7: 25-38.

15 Rodin E, Schmaltz S. The Bear-Fedio personality inventory and temporal lobe epilepsy. Neurology 1984;34:591-6.

16 American Psychiatric Association. Diagnostic and statistical manual of mental disorders. Washington DC: American Psychiatric Association, 1987.

17 Oldfield RC. The assessment and analysis of handedness: the Edinburgh inventory. Neuropsychologia 1971;9:97-113.

18 Cronbach LJ, Rajaratnam N, Gleser GC. Theory of generalizability: a liberalization of reliability theory. $B r J$ Stat Psychol 1963;16:137-63.

19 Goodwin C. Conversational organization: interaction between speakers and hearers. New York: Academic Press, 1981.

20 Clark HH, Schaefer EF. Collaborating on contributions to conversations. Lang Cogn Process 1987;2:19-41.

21 Bayles KA, Kaszniak AW, Tomoeda CK. Communication and cognition in normal aging and dementia. Boston: College-Hill Press, 1987:185-95.

22 Satz P. Pathological left-handedness: an explanatory model. Cortex 1972;8:121-35. 